\title{
1 A nucleus-like compartment shields bacteriophage DNA \\ 2 from CRISPR-Cas and restriction nucleases
}

3

4 Senén D. Mendoza ${ }^{1}$, Joel D. Berry ${ }^{1}$, Eliza S. Nieweglowska ${ }^{2}$,

5 Lina M. Leon ${ }^{1}$, David A. Agard ${ }^{2,3}$, Joseph Bondy-Denomy ${ }^{1,3 *}$

6

7 'Department of Microbiology and Immunology, University of California San Francisco, CA

894143, USA

$9{ }^{2}$ Howard Hughes Medical Institute (HHMI) and the Department of Biochemistry and Biophysics,

10 University of California San Francisco, San Francisco, CA 94158, USA

$11{ }^{3}$ Quantitative Biosciences Institute, University of California San Francisco, CA 94143, USA

12

13 *Correspondence: joseph.bondy-denomy@ucsf.edu 
14 All viruses require strategies to inhibit or evade the immunity pathways of cells they

15 infect. The viruses that infect bacteria, bacteriophages (phages), must avoid nucleic-acid

16 targeting immune pathways such as CRISPR-Cas and restriction endonucleases to

17 replicate efficiently ${ }^{1}$. Here, we show that a jumbo phage infecting Pseudomonas

18 aeruginosa, phage $\Phi K Z$, is resistant to many immune systems in vivo, including CRISPR-

19 Cas3 (Type I-C), Cas9 (Type II-A), Cas12 (Cpf1, Type V-A), and Type I restriction-

20 modification (R-M) systems. We propose that $\Phi K Z$ utilizes a nucleus-like shell to protect

21 its DNA from attack. Supporting this, we demonstrate that Cas9 is able to cleave $\Phi K Z$

22 DNA in vitro, but not in vivo and that Cas9 is physically occluded from the shell

23 assembled by the phage during infection. Moreover, we demonstrate that the Achilles

24 heel for this phage is the mRNA, as translation occurs outside of the shell, rendering the

25 phage sensitive to the RNA targeting CRISPR-Cas enzyme, Cas13a (C2c2, Type VI-A).

Collectively, we propose that the nucleus-like shell assembled by jumbo phages enables potent, broad spectrum evasion of DNA-targeting nucleases.

Phage infection and replication can cause bacterial death via cell lysis, necessitating protective immune systems to negate this great threat to bacterial viability ${ }^{2,3}$. Restriction-modification ( $R$ $\mathrm{M})$ and adaptive CRISPR-Cas (clustered regularly interspaced short palindromic repeats and CRISPR-associated genes) immunity detect and degrade phage nucleic acids to protect the host $^{1}$. Recent CRISPR-Cas discovery efforts have led to the characterization of six distinct CRISPR-Cas types (I-VI) with independent mechanisms for CRISPR RNA (crRNA) biogenesis, surveillance complex assembly, substrate selection, and target degradation ${ }^{4}$. Type III and VI CRISPR-Cas systems target RNA ${ }^{5,6}$, while Types I, II, and V predominantly target DNA ${ }^{7-9}$. Despite these differences, all characterized CRISPR-Cas systems function via crRNA-guides derived from the DNA-based CRISPR array, which stores the memory of past infections ${ }^{10,11}$.

Phages that infect Pseudomonas aeruginosa avoid CRISPR-mediated destruction by encoding "anti-CRISPR" (Acr) proteins that inhibit the Type I-E and I-F CRISPR-Cas systems ${ }^{12-14}$. We sought to determine whether any $P$. aeruginosa phages are also resistant to the Type I-C CRISPR-Cas subtype, also present in $P$. aeruginosa ${ }^{15}$. This subtype is notable as it is the most minimal Type I system identified to date, is highly abundant in bacterial genomes ${ }^{16}$, and is understudied relative to other subtypes. We identified an isolate in our lab encoding a Type I-C system, and designed and expressed a crRNA targeting related phages JBD30 and DMS3m, 
these phages by the Type I-C system demonstrated functional CRISPR immunity and that these phages did not possess functional Type I-C Acr proteins (Fig. 1a). To test the sensitivity of other phage families against the Type I-C system, we transferred the necessary cas genes (cas3, cas5, cas8, cas7) into the chromosome of a commonly used, phage-sensitive lab strain (PAO1) that naturally lacks CRISPR-Cas immunity. A small screen was then conducted, where crRNAs were tested against phages from five taxonomic groups: JBD30, D3, ФKZ, F8, and JBD68. JBD30, D3, and JBD68 are distinct temperate siphophages while $\Phi \mathrm{KZ}$ and F8 are distinct lytic myophages. All phages succumbed to targeting, except $\Phi K Z$ (Fig. 1b, 1c). $\Phi K Z$ titer did not decrease when exposed to ten different Type I-C crRNAs (Fig. 1c, Extended Data Fig. 1), suggesting that it is completely resistant to this immune system of $P$. aeruginosa.

The $\Phi K Z$ genome possesses no homologs of acr genes ${ }^{12-14,17,18}$ or anti-CRISPR associated (aca) genes that have previously enabled identification of acr genes ${ }^{14,17,18}$. Moreover, $\Phi K Z$ is a phage with no genetic tools currently available to manipulate it, rendering traditional knockout approaches infeasible. Thus to determine the mechanism by which the $\Phi K Z$ phage resists Type I CRISPR-Cas, we attempted to utilize the Type II-A CRISPR-Cas9 system from Streptococcus pyogenes (SpyCas9) to knock out phage genes. SpyCas9 and sgRNAs were adapted for expression and function in $P$. aeruginosa, leading to robust targeting of control phage JBD30, but notably, $\Phi \mathrm{KZ}$ replication and associated cell lysis was unaffected both in plate and liquid assays (Fig. 2a). An additional eight sgRNA sequences also failed to target $\Phi K Z$ (Extended Data Fig. 2a), suggesting that $\Phi K Z$ resists both Type I and II CRISPR immunity. Given the ability of this phage to evade two unrelated CRISPR systems originating from distantly related microbes, we considered that it may be generally resistant CRISPR-Cas immunity. To test this, the Type V-A Cas12a (Cpf1) CRISPR-Cas system from Moraxella bovoculi was expressed in $P$. aeruginosa and again, robust targeting of phage JBD30 was observed, but not of $\Phi \mathrm{KZ}$ with any of the nine crRNAs tested (Fig. 2b, Extended Data Fig. 2b). The ability of this phage to resist CRISPR systems found in its natural host (Type I-C) and those not present in Pseudomonas (Type II-A and V-A) suggests that this phage possesses a mechanism to enable "pan-CRISPR" resistance.

Restriction-modification systems are the most common bacterial immune system in nature and pose a significant impediment to phage replication ${ }^{1}$. To test whether $\Phi K Z$ is also resistant to attack from restriction endonucleases, the phage was propagated on strain PAK, an isolate that generates phages that are restricted by strain PAO1. When phage JBD30 was propagated on 
82 PAK, and then plated on strain PAO1, its titer was reduced by $\sim 3$ orders of magnitude (Fig. 2c),

83 an effect that was ameliorated in a PAO1 strain lacking the Type I R-M system $(\Delta h s d R)$.

84 However, when $\Phi K Z$ was propagated on PAK, its titer did not decrease on PAO1 (Fig. 2c).

85 Conversely, when propagated on PAO1, ФKZ titer was not decreased on PAK, while JBD30

86 was again reduced in titer by $\sim 3$ orders of magnitude (Fig. $2 \mathrm{c}$ ). This demonstrates that $\Phi K Z$ is

87 also recalcitrant to Type I restriction endonucleases in vivo.

Given the strong resistance to targeting, we considered whether DNA base modifications were protecting this phage from enzyme targeting, as has been previously seen with phage T4 and others $^{19-22}$. Purified phage DNA, extracted from $\Phi K Z$ virions, was subjected to restriction digestion reactions with a panel of restriction enzymes that are inhibited by glc-HmC moieties, including HindlII, EcoRI, Sacl, Kpnl, Ncol, and EcoRI (ref. 22,23 and per New England Biolabs). Additionally, MspJI was utilized, a modification-dependent restriction nuclease that requires 5$\mathrm{HmC}$ or 5-mC modified DNA for cleavage ${ }^{24}$. Except for Sacl, which lacks a sequence recognition motif in the $\Phi K Z$ genome, all enzymes tested cleaved $\Phi K Z$ gDNA, demonstrating the absence of glc-HmC modifications (Fig. 3a). To determine whether the phage genome is a substrate for CRISPR-Cas9-based cleavage, purified phage DNA was subjected to a cleavage assay with two distinct crRNA sequences using a dual crRNA:tracrRNA-loaded SpyCas9 nuclease in vitro. Cas 9 cleaved the phage genome in two locations, liberating the predicted 10 $\mathrm{kb}$ fragment from the much larger $280 \mathrm{~kb}$ genome (Fig. 3b). Notably, the two crRNA sequences used here are the same sequence as crRNAs II-A, \#1 and II-A, \#2 used in vivo (Fig. 2a),

103 demonstrating that these crRNA sequences do not target phage in vivo, but are competent for 104 genome cleavage in vitro.

Recently it was shown that $\Phi K Z$ and $\Phi K Z$-like phages infecting Pseudomonas sp. construct an

107 elaborate nucleus-like, proteinaceous compartment where phage DNA replicates, with PhuZ, a 108 phage tubulin homologue, centering the compartment within the host cell ${ }^{25-29}$. Proteins involved

109 in DNA replication and transcription localize inside the shell, while proteins mediating translation

110 and nucleotide synthesis are relegated to the cytoplasmic space, akin to the eukaryotic nucleus.

111 Given the rapid assembly of the shell and its apparent exclusion of select proteins, we

112 considered whether this structure was responsible for the resistance of $\Phi K Z$ to four unrelated

113 CRISPR-Cas and restriction nucleases. The Type II-A single protein effector Cas9 was chosen

114 as a representative immune system for ease of manipulation and imaging. $P$. aeruginosa cells

115 infected with $\Phi K Z$ were imaged with a Cas9 fluorescent antibody, revealing that the protein is 
116 indeed excluded from the shell during phage infection (Fig. 4a). DAPI staining reveals the phage

117 DNA inside the nucleus-like shell, while the host genome is rapidaly degraded during infection.

118 As a control, a protein previously shown to be internalized in the shell, ORF152, was imaged

119 revealing co-localization with the DAPI-positive phage nucleus. While the rules for protein

120 internalization in the shell are currently unknown, these data and work from the initial shell

121 studies ${ }^{28,29}$ suggest that the default localization for large host-encoded proteins is to be

122 excluded from the shell.

124 While ФKZ DNA is protected from CRISPR-Cas and restriction endonucleases, the mRNA is not 125 afforded this same luxury, as it leaves the confines of the shell to be translated in the cytoplasm.

126 We therefore envisaged that an RNA-targeting CRISPR-Cas system would provide immunity to

$127 \Phi K Z$ even if localized in the cytoplasm. To test this, we adapted the RNA-guided RNA nuclease

128 Cas13a (C2c2, Type VI-A) from Listeria seeligeri ${ }^{5,30}$ for phage targeting in $P$. aeruginosa. By

129 targeting several different transcripts, three LseCas13 spacers were found (two targeting tail

130 gene gp146 and one targeting the shell gene gp054) that decreased $\Phi K Z$ plaquing efficiency by

131 10-1000-fold (Fig. 4b, Extended Data Fig. 3). Corroborating the plaquing results, LseCas13 also

132 provided protection of $P$. aeruginosa in liquid cultures, with strong bacterial growth at phage

133 inputs that kill cultures with a non-targeting spacer (Fig. 4c). The sensitivity of $\Phi$ KZ to RNA-

134 targeting but not DNA-targeting underscores the role of the shell in broad spectrum resistance

135 to DNA-cleaving enzymes and also provides the first evidence of a DNA phage being inhibited

136 by Cas13.

138 Evasion of the endogenous $P$. aeruginosa Type I CRISPR-Cas system by $\Phi \mathrm{K} Z$ suggests that

139 these jumbo phages are likely to pervasively avoid this system in nature. Supporting this

140 hypothesis, our analysis of $>4,000 P$. aeruginosa non-redundant spacers (Type I-C, I-E, and I-F)

141 reported by van Belkum et al. (2015) found no spacers against $\Phi K Z$, or its jumbo phage

142 relatives ФPA3, PaBG, KTN4, and PA7 (Table 1). This is in contrast to the many spacer

143 matches from each system against diverse $P$. aeruginosa phages, such as those assayed in our

144 screen and those encoding anti-CRISPR proteins (Table 1). Finally, given the efficacy of the

145 RNA-targeting CRISPR-Cas13 system, we propose that perhaps these CRISPR systems are

146 well-suited to target the mRNA of DNA phages when the DNA is inaccessible (i.e. due to base

147 modifications or physical segregation). However, the rules governing the ability of Cas 13 to limit

148 the replication of DNA phages remain to be elucidated as only 3/11 LseCas 13a crRNAs tested

149 targeted $\Phi \mathrm{KZ}$ and 0/6 were effective at targeting phage JBD30 (Extended Data Fig. 3). 
151 Here, we searched for CRISPR-Cas resistant phages by designing crRNAs against them and

152 testing their efficacy. This screen identified jumbo phage $\Phi K Z$ (genome size: $280,334 \mathrm{bp}^{31}$ ) as

153 resistant to the Type I-C CRISPR system, and subsequently to Type II-A and Type V-A single

154 effector nucleases Cas9 and Cas12. $\Phi \mathrm{KZ}$ is also recalcitrant to the Type I restriction-

155 modification system of $P$. aeruginosa. Despite this apparent resistance in vivo, $\Phi \mathrm{KZ}$ genomic

156 DNA is sensitive to restriction enzymes and Cas9 cleavage in vitro. We propose that the

157 assembly of a proteinaceous compartment to house the replicating phage DNA creates a

158 physical protective barrier resulting in the resistance of phage $\Phi K Z$ to DNA-cleaving enzymes

159 (Fig. 4d). Although this shell-like structure has only been documented among the jumbo phages

160 of Pseudomonas ${ }^{28,29}$, we consider that physical occlusion of phage genomic DNA through this

161 and other mechanisms may comprise a novel route to immune system evasion in bacteria.

163 The pan-resistance of $\Phi K Z$ to DNA-targeting enzymes provides an explanation for the elaborate

164 and impressive shell structure, and suggests that the phage DNA may never be exposed to the

165 cytoplasm. Other hypotheses to describe the shell's existence remain to be addressed,

166 including protection from phage-derived nucleases that degrade the bacterial genome or as a

167 mechanism to spatially restrict the large phage genome during replication and packaging.

168 Regardless, we conclude that the nucleus-like shell provides a strong protective barrier to DNA-

169 targeting immune pathways. We expect that simple CRISPR-based screens, such as the one

170 conducted here, may reveal many other fascinating mechanisms that phages have evolved to

171 ensure their replicative success when faced with immune systems to overcome. 


\section{Acknowledgements:}

Research in the Bondy-Denomy lab was supported by the University of California San Francisco Program for Breakthrough in Biomedical Research, funded in part by the Sandler Foundation, and an NIH Office of the Director Early Independence Award (DP5-OD021344). This work was also supported by HHMI (DAA) and NIH grants R35GM118099 (DAA), and GM104556 (DAA).

$\Phi K Z$, JBD30, JBD68, D3, and F8 were provided by Alan Davidson's lab. Phage DMS3m was a gift from the O'Toole lab and Jason M. Peters and Carol A. Gross provided the S. pyogenes cas9 expression plasmid for integration in the PAO1 chromosome. pTE4495 (MbCpf1/MbCas12a) was a gift from Ervin Welker (Addgene plasmid \# 80339), and LseCas13a (Addgene plasmid \#83486) is a gift from Jennifer Doudna.

\section{Author Contributions:}

S.D.M. conducted restriction-modification experiments, constructed Cas 13 strains and conducted associated experiments with that strain and the Cas 12 strain, including all liquid infection assays, and prepared figures. J.D.B. constructed and conducted experiments with Cas 9 and Cas 12 expressing strains, and conducted in vitro digestion assays. L.M.L. conducted Type I-C Cas3 experiments. J.B.-D. conceived of the project, conducted Cas3 and Cas9 experiments, supervised all experiments, and wrote the manuscript together with S.D.M. E.S.N. conducted microscopy experiments, under the supervision of D.A.A. All authors edited the manuscript.

197 None to declare

200 Requests should be made to joseph.bondy-denomy@ucsf.edu 
Figures:
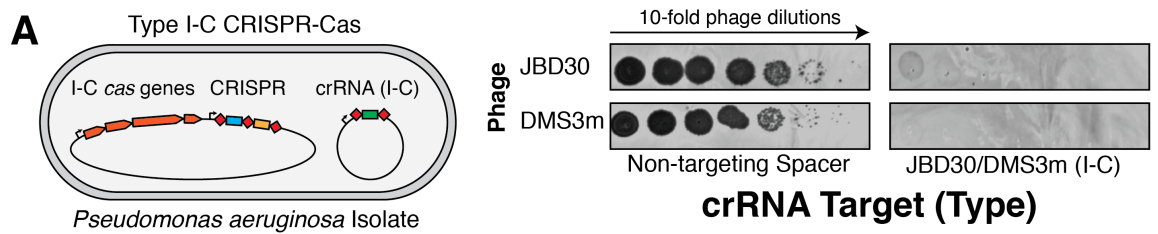

B
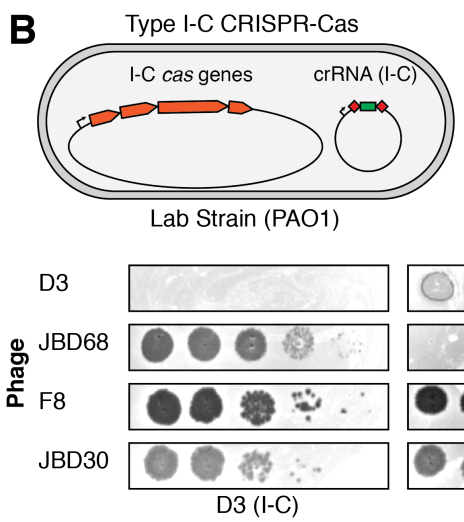

C
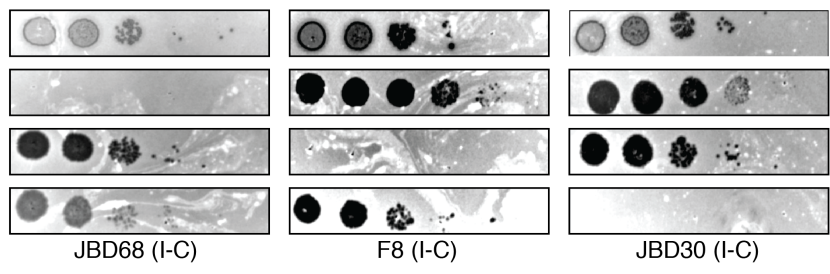

crRNA Target (Type)
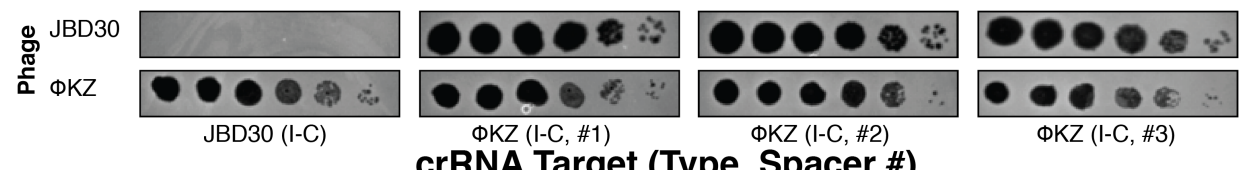

crRNA Target (Type, Spacer \#)

Figure 1: Identification of a phage that resists $P$. aeruginosa Type I-C CRISPR-Cas

immunity. a, Phages JBD30 and DMS3m were spotted in ten-fold serial dilutions on a lawn of a $P$. aeruginosa isolate naturally expressing the I-C cas genes, and an engineered crRNA to target both phages. Dark clearings in the lawn represent phage replication. b, Strain PAO1 was engineered to express the I-C cas genes and crRNAs targeting the indicated phages, and plaque assays were conducted as in Fig. 1a. c, PAO1 strains expressing crRNAs engineered to target phage JBD30 and phage $\Phi \mathrm{KZ}$ (I-C, \#1-\#3) were subjected to a plaque assay as in Fig. $1 \mathrm{a}$. 
A Type II-A CRISPR-Cas

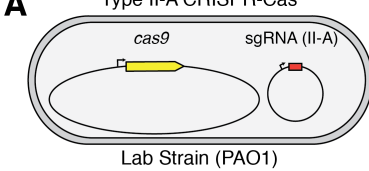

ФKZ Infections

1000 PFUs

- 100 PFUs

10 PFUs

$\triangle$ No Phage

Non-targeting Spacer

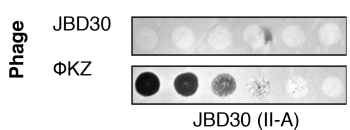

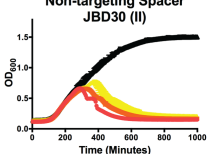

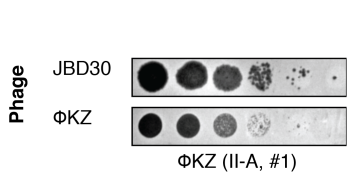

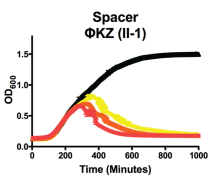

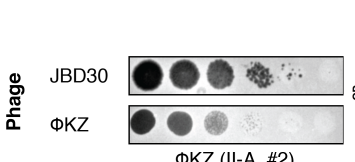
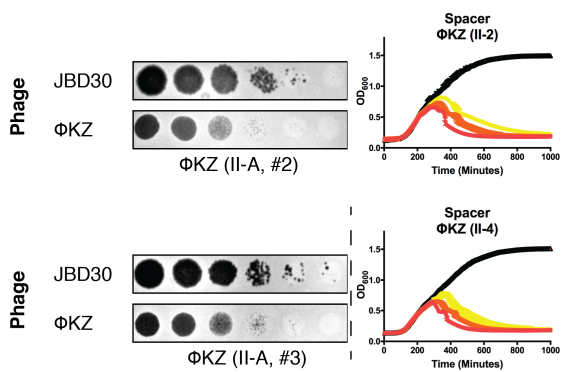

B

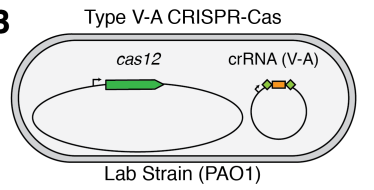

C

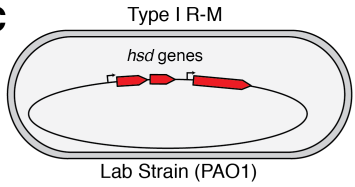

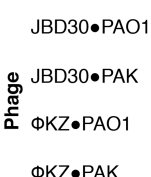

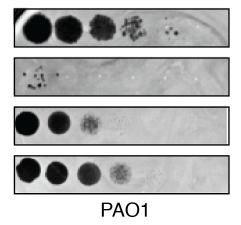

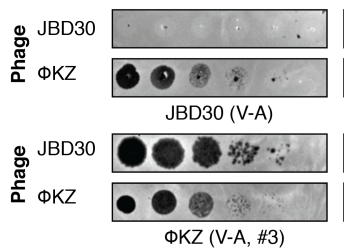

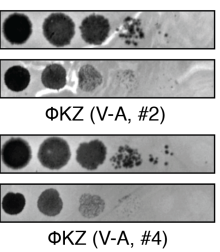

crRNA Target (Type, Spacer \#)

crRNA Target (Type, Spacer \#)

Figure 2: Jumbo phage $\Phi K Z$ resists targeting by heterologous Type II-A and V-A

CRISPR-Cas systems and and endogenous R-M system. a, Strain PAO1 was engineered to express the Type II-A Cas9 protein and single guide RNAs (sgRNAs) targeting the indicated phage. Plaque assays were conducted as in Figure 1a and growth curves were conducted, monitoring the OD600 of PAO1 cells infected with the indicated number of $\Phi K Z$ plaque forming units (pfu). b. Strain PAO1 was engineered to express the Type V-A Cas12a protein and crRNAs against the indicated phage. Plaque assays conducted as in Figure 1a. c, The endogenous Type I R-M system ( $h s d R S M$ ) in strains PAO1 and PAK was assayed using phages propoagated on PAO1 or PAK as indicated (e.g. JBD30•PAO1 was first propagated on strain PAO1). Together with an isogenic $\mathrm{PAO} 1 \Delta h s d R$ knockout, all strains were subjected to a plaque assay as in Figure 1a. 

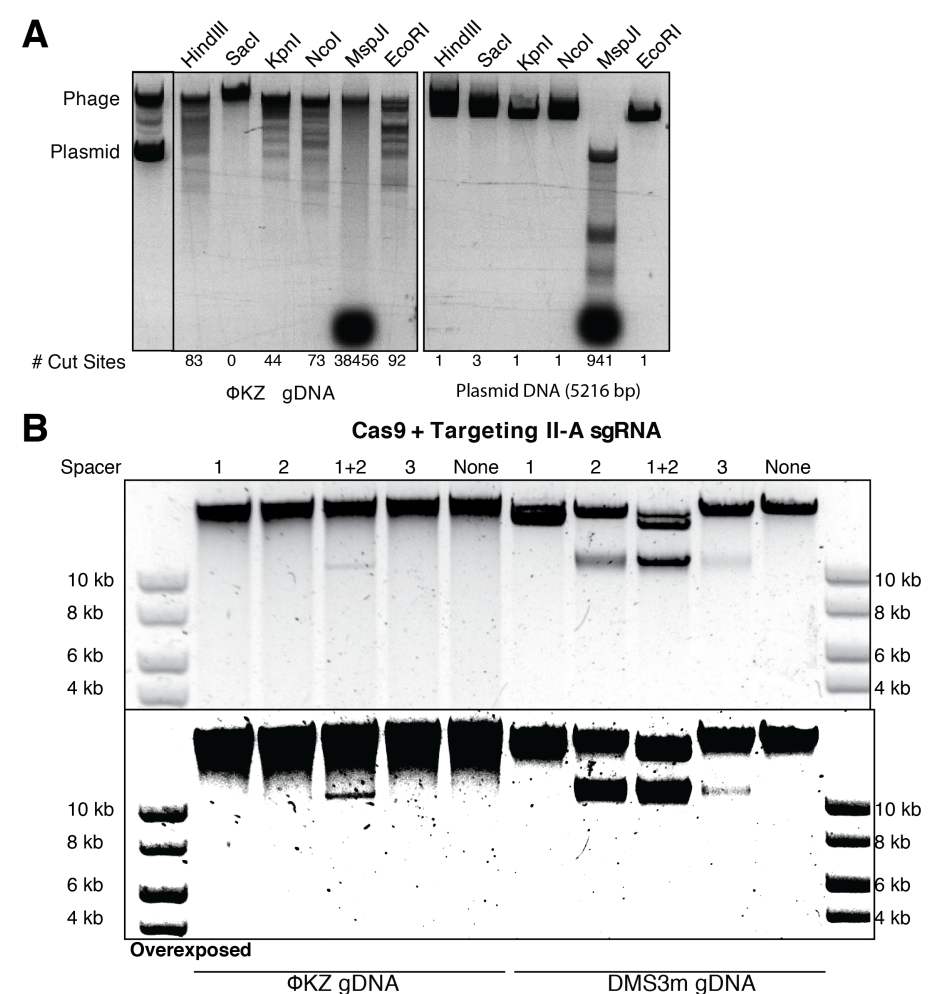

Figure 3: $\Phi K Z$ genomic DNA is sensitive to restriction enzymes and Cas9 in vitro. a, $\Phi K Z$ genomic DNA and plasmid DNA were subjected to digestion with the indicated restriction enzymes in vitro. The first lane contains purified phage and plasmid DNA run together. The number of cut sites for each enzyme is shown at the bottom of the gels. $\mathbf{b}, \Phi K Z$ and phage DMS3m genomic DNA were digested in vitro using Cas9 loaded with crRNA:tracrRNA targeting the indicated phage. The bottom gel is the same as the top gel, however the image was overexposed to enhance faint bands. Products were visualized on a $0.7 \%$ agarose gel, visualized with SYBR Safe nucleic acid stain. 
A
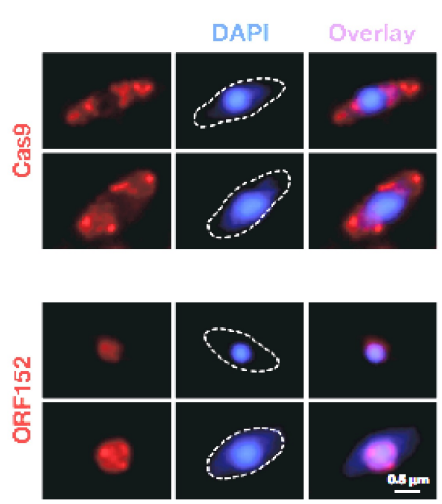

C

D

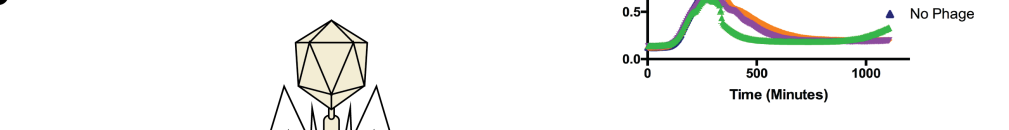

Type VI-A CRISPR-Cas
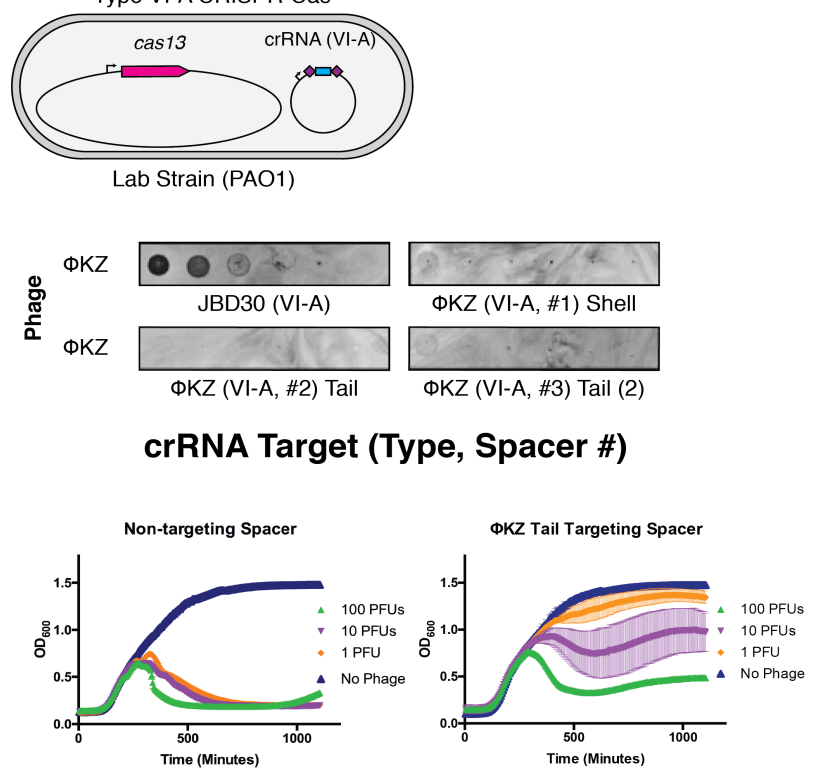

Figure 4: Phage $\Phi K Z$ DNA is protected from Cas9 and other DNA targeting enzymes, but is sensitive to RNA-targeting Cas13. a, Fluorescence microscopy of $P$. aeruginosa, immunostained for Cas9 (top panels), or Myc-ORF152 (bottom panels). DAPI stain shows the phage DNA within the shell. $\mathbf{b}$, Strain PAO1 expressing LseCas13a and a crRNA targeting the indicated phage. Plaque assays conducted as in Figure 1a. c, Growth curves measuring the OD600 of PAO1 infected with the indicated number of $\Phi K Z$ plaque forming units (PFUs). d, A model summarizing the $\Phi K Z$ nucleus-like shell excluding Cas9, Cas12, Cascade-Cas3 (Type I$\mathrm{C}$, and Type I restriction endonucleases (REase), while the mRNA (red) is exported and can be targeted by Cas 13. 
Tables:

Table 1: $\Phi K Z$ and $\Phi K Z$-like phages have no natural spacers matching their genomes from a natural collection of $>4000 P$. aeruginosa spacers.

253 The total number of Type I CRISPR spacers with a perfect match to the indicated P. aeruginosa phages assayed in this study and previous CRISPR-Cas studies. The experimental sensitivity of each phage to the indicated subtypes are shown. AcrIE3 and AcrIF1 are I-E and I-F antiCRISPR proteins, respectively. ${ }^{*}$ indicates that all spacers have mismatches $(\leq 4)$ to the F8 genome.

\begin{tabular}{|c|c|c|c|}
\hline Phage & \# spacers & Type I CRISPR sensitivity & Reference \\
\hline DMS3m & 75 & $\begin{array}{l}\text { I-C: sensitive } \\
\text { I-E: resistant (AcrIE3) } \\
\text { I-F: sensitive }\end{array}$ & This study, ref. 13,33 \\
\hline JBD30 & 51 & $\begin{array}{l}\text { I-C: sensitive } \\
\text { I-E: sensitive } \\
\text { I-F: resistant (AcrlF1) }\end{array}$ & This study, ref. 12,13 \\
\hline JBD18 & 51 & I-F: sensitive & Ref. 33 \\
\hline JBD25 & 46 & I-F: sensitive & Ref. 33 \\
\hline JBD68 & 28 & I-C: sensitive & This study \\
\hline D3 & 49 & I-C: sensitive & This study \\
\hline F8 & $3^{*}$ & I-C: sensitive & This study \\
\hline$\Phi \mathrm{KZ}$ & 0 & I-C: resistant & This study \\
\hline phiPA3 & 0 & not assayed & \\
\hline PaBG & 0 & not assayed & \\
\hline KTN4 & 0 & not assayed & \\
\hline PA7 & 0 & not assayed & \\
\hline
\end{tabular}




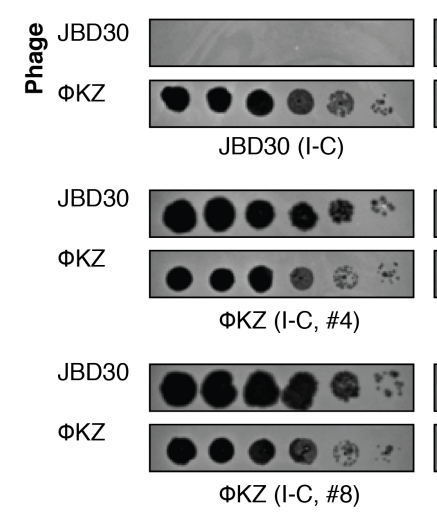
of the indicated PAO1 strain.
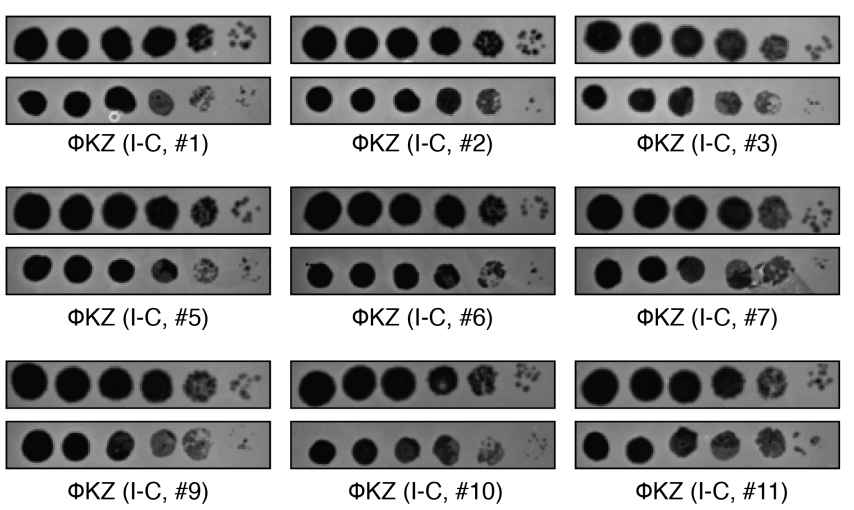

crRNA Target (Type, Spacer \#)

\section{Extended Data Figure 1: Phage $\Phi$ KZ is resistant to Type I-C CRISPR-Cas immunity.} $P$. aeruginosa strain PAO1 expressing the Type I-C cas genes and crRNAs programmed to recognize the indicated phage. One crRNA targeting JBD30 is shown, while 11 distinct crRNAs are directed towards $\Phi K Z$. Phages are spotted in ten-fold serial dilutions (left to right) on a lawn 
A

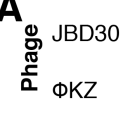
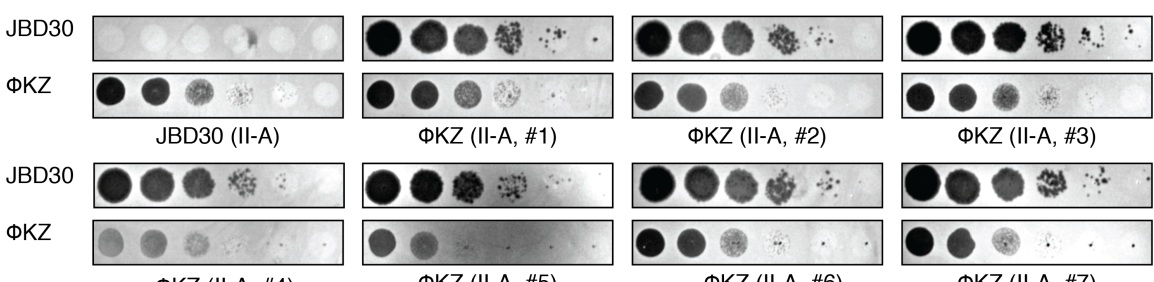

JBD30

ФKZ (II-A, \#4)

ФKZ (II-A, \#5)

ФKZ (II-A, \#6)

ФKZ (II-A, \#7)

$\Phi K Z$
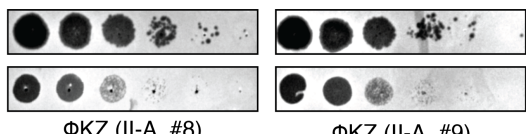

crRNA Target (Type, Spacer \#)
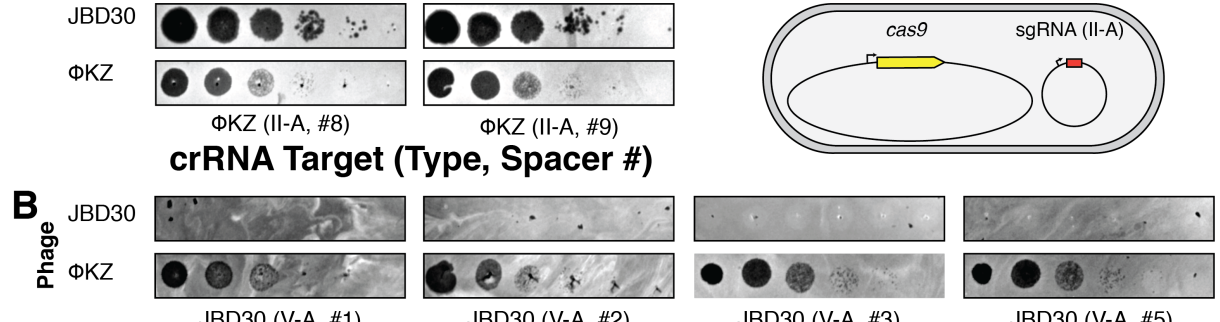

JBD30 (V-A, \#1)

JBD30 (V-A, \#2)
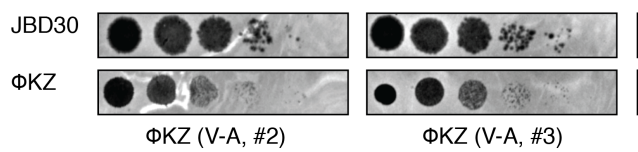

JBD30 (V-A, \#3)

JBD30 (V-A, \#5)

JBD30

$\Phi K Z$

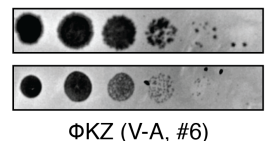

JBD30 ФKZ (V-A, \#6)
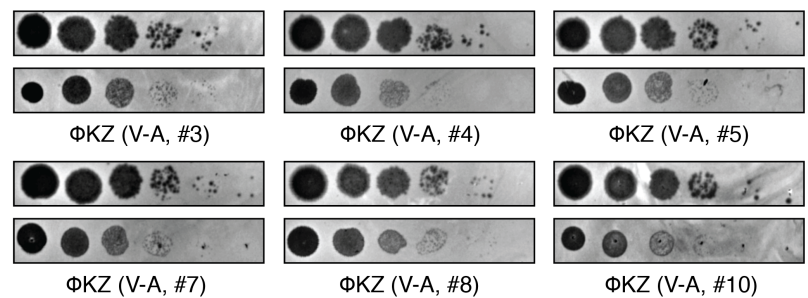

$\Phi K Z$

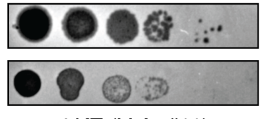

ФKZ (V-A,\#11)

crRNA Target (Type, Spacer \#)

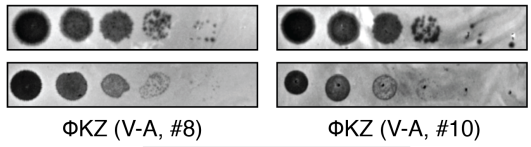

Extended Data Figure 2: Phage $\Phi K Z$ is resistant to Type II-A and V-A CRISPR-Cas immunity. $P$. aeruginosa strain PAO1 expressing the (a) Type II-A Cas9 or (b) Type V-A Cas12a effectors and sgRNAs/crRNAs programmed to recognize the indicated phage. Phages are spotted in ten-fold serial dilutions (left to right) on a lawn of the indicated PAO1 strain. 
A
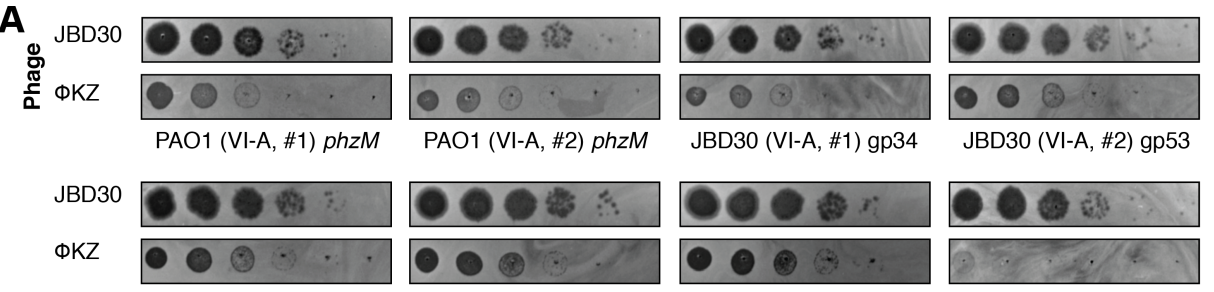

JBD30
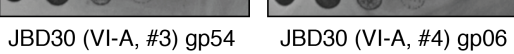

JBD30 (VI-A, \#5) gp07

ФKZ (VI-A, \#1) gp054

$\Phi K Z$
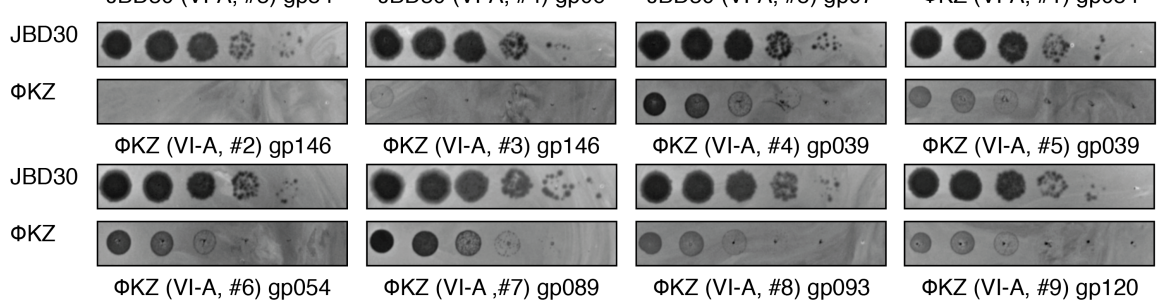

ФKZ (VI-A, \#3) gp146
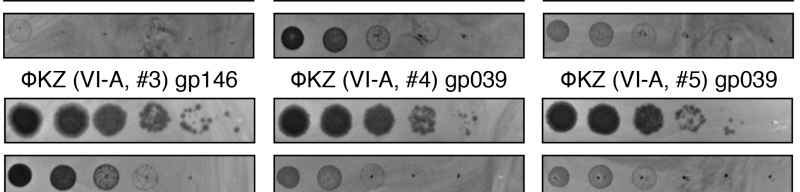

ФKZ (VI-A, \#5) gp039

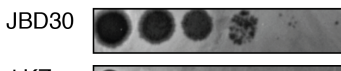

ФKZ (VI-A ,\#7) gp089

ФKZ (VI-A, \#8) gp093

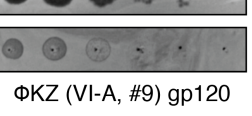

$\Phi K Z$
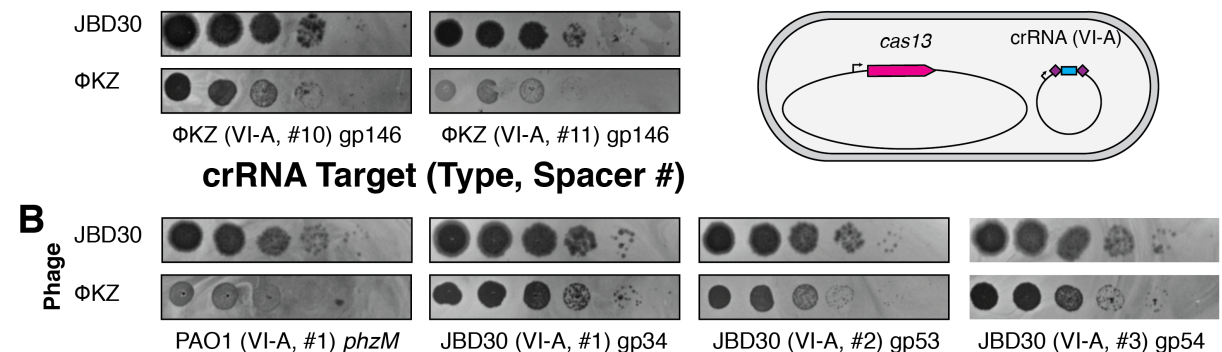

PAO1 (VI-A, \#1) phzM

JBD30 (VI-A, \#1) gp34
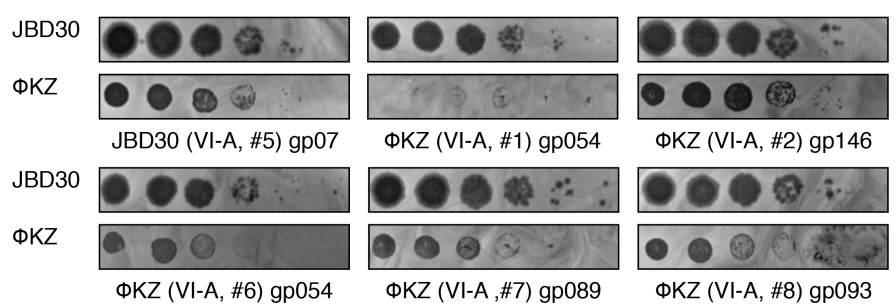

JBD30 (VI-A, \#3) gp54
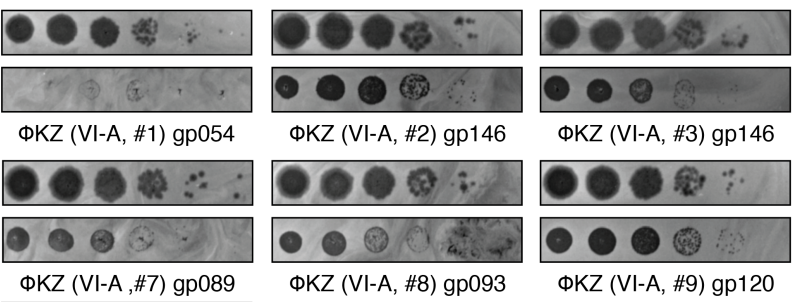

JBD30

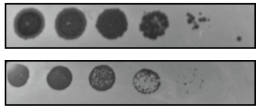

ФKZ (VI-A ,\#7) gp089

$\Phi K Z$

ФKZ (VI-A, \#10) gp146

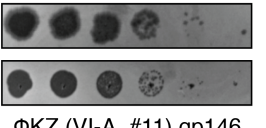

crRNA Target (Type, Spacer \#)

Extended Data Figure 3: Phage $\Phi K Z$ is sensitive to Type VI-A CRISPR-Cas immunity. $P$. aeruginosa strain PAO1 expressing the Type VI-A (a) LseCas13a or (b) LshCas13a effectors and crRNAs programmed to recognize the indicated phage. Phages are spotted in ten-fold serial dilutions (left to right) on a lawn of the indicated PAO1 strain. 


\section{Methods}

\section{Bacterial growth and genetic manipulation}

281 Strains, plasmids, phages, and spacer sequences used in this study are listed in Supplemental

282 Tables 1-4. Pseudomonas aeruginosa strain PAO1 was grown in LB at $37{ }^{\circ} \mathrm{C}$ with aeration at

283225 RPM. When necessary plating was performed on LB agar with carbenicillin $(200 \mu \mathrm{g} / \mathrm{ml})$ or

284 gentamicin $(50 \mu \mathrm{g} / \mathrm{ml})$. Gene expression was induced by the addition of L-arabinose $(0.1 \%$ final $)$

285 and/or isopropyl $\beta$-D-1-thiogalactopyranoside (IPTG, $0.5 \mathrm{mM}$ or $1 \mathrm{mM}$ final). For chromosomal

286 insertions at the attTn7 locus, $P$. aeruginosa cells were electroporated with the integrating

287 vector pUC18T-lac and the transposase expressing helper plasmid pTNS3, and selected on

288 gentamicin. Potential integrants were screened by colony PCR with primers PTn7R and PgImS-

289 down. Electrocompetent cell preparations, transformations, integrations, selections, plasmid

290 curing, and FLP recombinase mediated marker excision with pFLP were performed as

291 described previously ${ }^{32}$.

\section{Phage growth and DNA extraction}

294 Phage growth was conducted in LB at $37^{\circ} \mathrm{C}$ with PAO1 as a host. Growth curves were

295 conducted in a Biotek Synergy plate reader at $37^{\circ} \mathrm{C}$ with orbital shaking set to maximum speed.

296 Phage stocks were diluted and stored in SM buffer ${ }^{33}$ and used for routine plaquing assays. For

297 high titer lysates to generate phage DNA, plates with a high number of plaques were flooded

298 with SM buffer and collected ${ }^{33}$. The lysates were subsequently DNase treated and filtered

299 through a $0.22 \mu \mathrm{m}$ filter. Phage DNA was extracted with the Wizard Genomic DNA Purification

300 Kit (Promega). DNA restriction assays were performed according to standard NEB protocols

301 and restriction fragments were assessed by agarose gel electrophoresis.

\section{Type I-C CRISPR-Cas system expression in $P$. aeruginosa PA01}

304 Type I-C CRISPR-Cas function was tested by electroporating a strain containing I-C cas genes

305 with pHERD30T plasmids encoding crRNAs that target phages. To express this system

306 heterologously in PAO1, the four effector cas genes (cas3-5-8-7) were cloned into pUC18T-lac

307 and inserted in the PAO1 chromosome as described above. After removal of the gentamicin

308 marker, this strain was electroporated with the same pHERD30T crRNA-encoding plasmids to

309 confirm function upon IPTG/arabinose induction. 
312 All crRNAs used here, were cloned into established entry vectors in the pHERD30T

313 background. After removing a pre-existing Bsal cut site in the vector by mutagenesis, a pseudo-

314 CRISPR array (i.e. repeat-spacer-repeat for Type I, V, VI, or a sgRNA scaffold for Type II) was

315 then cloned into the vector, where the spacing sequence possessed two inverted Bsal digest

316 sites, to facilitate scarless cloning of new spacers. Desired spacer sequences were ordered as

317 two complementary oligonucleotides that generate sticky ends when annealed, to be cloned into

318 the entry vector, which was Bsal digested. Spacer oligonucleotides were PNK treated,

319 annealed, and ligated into the entry vector.

\section{Streptococcus pyogenes (Spy) Cas 9 and sgRNA expression in $\boldsymbol{P}$. aeruginosa}

322 The S. pyogenes Cas9 gene was cloned into a pUC18T-Ara integration vector and then inserted

323 into the attTn7 locus of PAO1. A single guide RNA scaffold was constructed based on a

324 previous design ${ }^{34}$ with internal Bsal cut sites to enable insertion of pre-annealed oligos for

325 scarless sgRNA design. This sgRNA scaffold was amplified with primers p30T-gRNA_Bsal and

326 p30T-gRNA_Bsal_rev. The resulting product was inserted into the pHERD30T vector via

327 Gibson assembly following backbone (pJW1) amplification by inverse PCR with primers

328 gRNA_Bsal-p30T and gRNA_Bsal-p30T-rev. The sgRNA scaffold was positioned into pJW1 so

329 that following Bsal cleavage the spacer insert +1 position would conincide with the pBAD TSS

$330+1$ position. The resulting plasmid, pJB1, was Bsal digested (NEB) followed by ligation of

331 indicated pre-annealed oligos. Table 3 contains a complete list of all target sequences. The

332 sequence of the sgRNA construct with Bsal site locations is shown in Supplemental Table 3.

\section{Cas9 in vitro cleavage}

335 Cas9-based phage genome cleavage in vitro was conducted with purified Cas9 protein (NEB

336 \#M0386S), and the Cas9-gRNA-tracrRNA based cleavage reaction was then performed using

337 according to the manufacturer's (NEB) instructions. Cas9 crRNAs (Supplemental Table 3) were

338 ordered as Alt-R CRISPR-Cas 9 crRNAs from IDT and utilized without further modification. The

339 tracrRNA was amplified using primers tracrRNA-FOR and tracrRNA-REV from a plasmid

340 (pBR62). The tracrRNA was produced through a T7 RNAP reactions using dsDNA encoding the

341 tracrRNA downstream of a T7 RNAP promoter. Cas9 protein (NEB) was combined with pre-

342 annealed crRNA and tracRNA complex at a 1:1 molar ratio. The reaction was performed at 37

$343{ }^{\circ} \mathrm{C}$ for $4 \mathrm{hrs}$ with $300 \mathrm{ng}$ of $\Phi \mathrm{KZ}$ or DMS3 genomic DNA and the products were assessed by

344 agarose gel electrophoresis. Two Cas9 guides were selected that would cleave at pos. 158,649

345 and 168,715 of the $\Phi K Z$ genome to liberate an $\sim 10 \mathrm{~kb}$ fragment. 


\section{Cas12a and crRNA design for PA expression}

348 The humanized allele of the cpf1 gene of Moraxella bovoculli (MBO_03467, KDN25524.1) was

349 sub-cloned from pTE4495 (Addgene) into pUC18T-lac using primers pUC_cpf1_F and

350 pUC_cpf1_R and inserted in the PAO1 chromosome as described above. A Cpf1 repeat-

351 spacer-repeat pseudo-CRISPR array was synthesized as oligonucleotides, annealed, and

352 ligated into a pHERD30T vector, digested with $\mathrm{Ncol}$ and HindIII. Spacer sequences were cloned

353 into the resulting vector (pJB2) following Bsal digestion and ligation of pre-annealed spacer

354 oligonucleotide pairs.

\section{Cas13a and crRNA design for PA expression}

357 The wild type allele of the cas13 gene of Listeria seeligeri and Leptotrichia shahii were subcloned from p2CT-His-MBP-Lse_C2c2_WT and p2CT-His-MBP-Lsh_C2c2_WT (Addgene) into pUC18T-lac. LseCas13 and Lsh Cas13 were inserted in the PAO1 chromosome as described above. An Lse and an Lsh Cas13a repeat-spacer-repeat pseudo-CRISPR array were synthesized as oligonucleotides, annealed, and ligated into a pHERD30T vector, digested with agar, $10 \mathrm{mM} \mathrm{MgSO}_{4}, 1 \mathrm{mM}$ IPTG molten top agar were seeded with $100 \mu \mathrm{L}$ saturated culture and spread on $20 \mathrm{~mL} 10 \mathrm{mM} \mathrm{MgSO}_{4}, 50 \mu \mathrm{g} / \mathrm{mL}$ gentamicin, 0.1\% (L)-arabinose, $1 \mathrm{mM}$ IPTG LB agar plates. $2.5 \mu \mathrm{L} 10$-fold serial dilutions of bacteriophage JBD30 and $\Phi K Z$ were spotted on plates. Plates were incubated at $37^{\circ} \mathrm{C}$ overnight and were imaged on the following day.

\section{Restriction-Modification Assay}

372 The PAO1 hsdR gene (PA2732) was knocked out using CRISPR-Cas9 and a targeted sgRNA. 373 PAO1, PAK, and PAO1 $\Delta h s d R$ were grown to saturation in LB at $37^{\circ} \mathrm{C} .4 \mathrm{~mL}$ of $0.7 \%$ agar, 10 $374 \mathrm{mM} \mathrm{MgSO}_{4}$ molten top agar were seeded with $100 \mu \mathrm{L}$ saturated culture and spread on $20 \mathrm{~mL}$ $37510 \mathrm{mM} \mathrm{MgSO}_{4}$ LB agar plates. $2.5 \mu \mathrm{L}$ 10-fold serial dilutions of bacteriophage JBD30 and ФKZ 376 propagated on strain PAO1 and PAK were spotted on plates. Plates were incubated at $37^{\circ} \mathrm{C}$ 377 overnight and were imaged the following day. 


\section{Sample Growth}

$3815 \mathrm{~mL}$ overnight cultures of a strain expressing Cas9 and an sgRNA targeting ФKZ (SDM065)

382 and a strain expressing cMyc-ORF152 (bESN27) were grown at $30^{\circ} \mathrm{C}$ in LB media with

383 gentamicin. A 1:30 back-dilution of the overnight culture into LB was grown at $30{ }^{\circ} \mathrm{C}$ for $1 \mathrm{~h}$.

384 Protein and guide expression was induced with $0.1 \%$ arabinose and $0.5 \mathrm{mM} \mathrm{IPTG}$, respectively.

385 After $1 \mathrm{~h}$ of expression, an aliquot of uninfected cells was fixed while the remaining cultures

386 were infected with $\Phi K Z$ using MOI 1.5. Infected cell aliquots were collected and fixed at 60 mpi.

388 Fixation

389 This protocol was adapted from ref. 35. Samples were fixed with 5X Fix Solution (12.5\%

390 paraformaldehyde, $150 \mathrm{mM} \mathrm{KPO4,} \mathrm{pH}$ 7.2) and incubating for 15 minutes at room temperature

391 followed by 20 minutes on ice. Samples were then washed in PBS 3 times and finally

392 resuspended in GTE (50 mM glucose, 10 mM EDTA, pH 8.0, 20 mM Tris- $\mathrm{HCl}, \mathrm{pH} 7.65$ ) with 10

$393 \mathrm{ug} / \mathrm{mL}$ lysozyme. Resuspended cells were transferred to polylysinated coverslips and dried.

394 Once dry, coverslips were incubated in cold methanol for 5 minutes followed by cold acetone for

3955 minutes. Cells were rehydrated by a rinse in PBS followed by a 3-minute incubation in PBS +

$3962 \%$ BSA blocking solution. Cells were incubated with a 1:50 dilution of primary antibody (Cas9

397 (7A9-3A3): sc-517386 or cMyc (9E10): sc-40) in PBS + 2\% BSA for $1 \mathrm{hr}$ followed by 3,7 minute

398 washes in fresh PBS + 2\% BSA. Coverslips were then incubated in the dark for $1 \mathrm{hr}$ with

399 secondary antibody (goat anti-mouse Alexa Fluor 555, Life Technologies A-21424) diluted 1:500

400 in PBS $+2 \%$ BSA. DAPI was added for the final 10 minutes of the incubation. Cells were

401 washed in PBS 3 times for 7 minutes. Coverslips were then placed on slides using mounting

402 media ( $/ / v 90 \%$ glycerol, v/v 10\% Tris $\mathrm{pH} 8.0$ and w/v 0.5\% propyl-gallate) and sealed with clear

403 nail polish.

404

405 Microscopy and Analysis

406 Images were collected using a Zeiss Axiovert 200M microscope. 


\section{REFERENCES}

408 1. Koonin, E. V., Makarova, K. S. \& Wolf, Y. I. Evolutionary Genomics of Defense Systems in Archaea and Bacteria. Annu Rev Microbiol 71, annurev-micro-090816-093830 (2017).

2. Labrie, S. J., Samson, J. E. \& Moineau, S. Bacteriophage resistance mechanisms. Nat Rev Micro 8, 317-327 (2010).

3. Doron, S. et al. Systematic discovery of antiphage defense systems in the microbial pangenome. Science 359, eaar4120 (2018).

4. Hille, F. et al. The Biology of CRISPR-Cas: Backward and Forward. Cell 172, 1239-1259 (2018).

5. Abudayyeh, O. O. et al. C2c2 is a single-component programmable RNA-guided RNAtargeting CRISPR effector. Science 353, aaf5573 (2016).

6. Hale, C. R. et al. RNA-Guided RNA Cleavage by a CRISPR RNA-Cas Protein Complex. Cell 139, 945-956 (2009).

7. Mulepati, S. \& Bailey, S. Structural and biochemical analysis of nuclease domain of clustered regularly interspaced short palindromic repeat (CRISPR)-associated protein 3 (Cas3). J Biol Chem 286, 31896-31903 (2011).

8. Garneau, J. E. et al. The CRISPR/Cas bacterial immune system cleaves bacteriophage and plasmid DNA. Nature 468, 67-71 (2010).

9. Zetsche, B. et al. Cpf1 Is a Single RNA-Guided Endonuclease of a Class 2 CRISPR-Cas System. Cell 163, 759-771 (2015).

10. Barrangou, R. et al. CRISPR provides acquired resistance against viruses in prokaryotes. Science 315, 1709-1712 (2007).

11. Brouns, S. J. J. et al. Small CRISPR RNAs guide antiviral defense in prokaryotes. Science 321, 960-964 (2008).

12. Bondy-Denomy, J., Pawluk, A., Maxwell, K. L. \& Davidson, A. R. Bacteriophage genes that inactivate the CRISPR/Cas bacterial immune system. Nature 493, 429-432 (2013).

13. Pawluk, A., Bondy-Denomy, J., Cheung, V. H. W., Maxwell, K. L. \& Davidson, A. R. A new group of phage anti-CRISPR genes inhibits the type I-E CRISPR-Cas system of Pseudomonas aeruginosa. mBio 5, e00896-e00896-14 (2014).

14. Pawluk, A. et al. Inactivation of CRISPR-Cas systems by anti-CRISPR proteins in diverse bacterial species. Nature Microbiology 1, 1-6 (2016).

15. van Belkum, A. et al. Phylogenetic Distribution of CRISPR-Cas Systems in AntibioticResistant Pseudomonas aeruginosa. mBio 6, e01796-15 (2015).

16. Makarova, K. S. et al. An updated evolutionary classification of CRISPR-Cas systems. Nat Rev Micro 13, 722-736 (2015).

17. Pawluk, A. et al. Naturally Occurring Off-Switches for CRISPR-Cas9. Cell 167, 18291838.e9 (2016).

18. Rauch, B. J. et al. Inhibition of CRISPR-Cas9 with Bacteriophage Proteins. Cell 168, 150-158.e10 (2017).

19. Bryson, A. L. et al. Covalent Modification of Bacteriophage T4 DNA Inhibits CRISPRCas9. mBio 6, e00648-15-9 (2015).

20. Strotskaya, A. et al. The action of Escherichia coli CRISPR-Cas system on lytic bacteriophages with different lifestyles and development strategies. Nucleic Acids Research 45, 1946-1957 (2017).

21. Vlot, M. et al. Bacteriophage DNA glucosylation impairs target DNA binding by type I and II but not by type V CRISPR-Cas effector complexes. Nucleic Acids Research 46, 873885 (2018).

22. Huang, L. H., Farnet, C. M., Ehrlich, K. C. \& Ehrlich, M. Digestion of highly modified bacteriophage DNA by restriction endonucleases. Nucleic Acids Research 10, 1579- 
1591 (1982).

23. Kaplan, D. A. \& Nierlich, D. P. Cleavage of Nonglucosylated Bacteriophage T4 deoxyribonucleic acid by Restriction Endonuclease Eco RI. J Biol Chem 250, 2395-2397 (1975).

24. Cohen-Karni, D. et al. The MspJI family of modification-dependent restriction endonucleases for epigenetic studies. Proceedings of the National Academy of Sciences 108, 11040-11045 (2011).

25. Kraemer, J. A. et al. A phage tubulin assembles dynamic filaments by an atypical mechanism to center viral DNA within the host cell. Cell 149, 1488-1499 (2012).

26. Erb, M. L. et al. A bacteriophage tubulin harnesses dynamic instability to center DNA in infected cells. Elife 3, e03197 (2014).

27. Zehr, E. A. et al. The structure and assembly mechanism of a novel three-stranded tubulin filament that centers phage DNA. Structure 22, 539-548 (2014).

28. Chaikeeratisak, V. et al. Assembly of a nucleus-like structure during viral replication in bacteria. Science 355, 194-197 (2017).

29. Chaikeeratisak, V. et al. The Phage Nucleus and Tubulin Spindle Are Conserved among Large Pseudomonas Phages. Cell Rep 20, 1563-1571 (2017).

30. Gootenberg, J. S. et al. Nucleic acid detection with CRISPR-Cas13a/C2c2. Science 356, eaam9321-442 (2017).

31. Mesyanzhinov, V. V. et al. The genome of bacteriophage phi KZ of Pseudomonas aeruginosa. Journal of Molecular Biology 317, 1-19 (2002).

32. Choi, K.-H. \& Schweizer, H. P. mini-Tn7 insertion in bacteria with single attTn7 sites: example Pseudomonas aeruginosa. Nat Protoc 1, 153-161 (2006).

33. Cady, K. C., Bondy-Denomy, J., Heussler, G. E., Davidson, A. R. \& O'Toole, G. A. The CRISPR/Cas adaptive immune system of Pseudomonas aeruginosa mediates resistance to naturally occurring and engineered phages. 194, 5728-5738 (2012).

34. Jinek, M. et al. A Programmable Dual-RNA-Guided DNA Endonuclease in Adaptive Bacterial Immunity. Science 337, 816-821 (2012).

35. Cowles, K. N. et al. The putative Poc complex controls two distinct Pseudomonas aeruginosa polar motility mechanisms. Molecular Microbiology 90, 923-938 (2013).

36. Bondy-Denomy, J. et al. Prophages mediate defense against phage infection through diverse mechanisms. The ISME Journal 10, 2854-2866 (2016).

37. Kropinski, A. M. Sequence of the genome of the temperate, serotype-converting, Pseudomonas aeruginosa bacteriophage D3. 182, 6066-6074 (2000).

38. Qiu, D., Damron, F. H., Mima, T., Schweizer, H. P. \& Yu, H. D. PBAD-Based Shuttle Vectors for Functional Analysis of Toxic and Highly Regulated Genes in Pseudomonas and Burkholderia spp. and Other Bacteria. Applied and Environmental Microbiology 74, 7422-7426 (2008).

39. Choi, K.-H. \& Schweizer, H. P. mini-Tn7 insertion in bacteria with single attTn7 sites: example Pseudomonas aeruginosa. Nat Protoc 1, 153-161 (2006).

40. Choi, K.-H. et al. Genetic tools for select-agent-compliant manipulation of Burkholderia pseudomallei. Applied and Environmental Microbiology 74, 1064-1075 (2008). 
Supplementary Information

502 Strains, Plasmids, and Oligonucleotides

503 Supplemental Table 1. Phage and Strains

504

\begin{tabular}{|l|l|l|}
\hline Name & Source & Reference \\
\hline ФKZ & Davidson Lab & 31 \\
\hline DMS3m & O'Toole Lab & 33 \\
\hline JBD30 & Davidson Lab & 36 \\
\hline F8 & Davidson Lab & $\begin{array}{l}\text { Unpublished } \\
\text { Accession: DQ163917 }\end{array}$ \\
\hline D3 & Davidson Lab & 37 \\
\hline JBD68 & Davidson Lab & 36 \\
\hline & & \\
\hline & Genotype & Reference \\
\hline JW31 & PAO1 tn7::I-C/Cas3 $3^{\text {PA }}$ & This study \\
\hline JB10 & PAO1 tn7::cas9 ${ }^{\text {Spy }}$ & This study \\
\hline JB90 & PAO1 tn7::cas12a & This study \\
\hline SDM084 & PAO1 tn7:::cas13 $13^{\text {Le }}$ & This study \\
\hline SDM020 & PAO1 $\triangle h s d R$ & This study \\
\hline
\end{tabular}

505

506

507

Supplemental Table 2. Plasmids
\begin{tabular}{|l|l|l|}
\hline Name & Information & Ref. \\
\hline pHERD30T & $\begin{array}{l}\text { Arabinose inducible, gentamicin } \\
\text { resistant shuttle vector }\end{array}$ & 38 \\
\hline pUC18T-Lac & $\begin{array}{l}\text { IPTG inducible, Amp }{ }^{R} / \text { Gent } \\
\text { integrative plasmid with FRT sites } \\
\text { integranking gentamicin cassette }\end{array}$ & 39 \\
\hline pTNS3 & $\begin{array}{l}\text { Expresses tnsABCD for flipping out } \\
\text { cassette flanked by FRT sites }\end{array}$ & 40 \\
\hline pBR62 & $\begin{array}{l}\text { Contains Spy Cas9 tracrRNA } \\
\text { sequence }\end{array}$ & This study \\
\hline pJW1 & $\begin{array}{l}\text { pHERD30T with Bsal site at pos. 235 } \\
\text { GAGACC mutated to GTGACC }\end{array}$ & This study \\
\hline pJW13 & $\begin{array}{l}\text { pJW1 with Type I-C pseudo-CRISPR } \\
\text { array for spacer cloning }\end{array}$ & This study \\
\hline pJB1 & $\begin{array}{l}\text { pJW1 with Type II-A sgRNA backbone } \\
\text { at the +1 TSS of pBAD }\end{array}$ & This study \\
\hline pJB2 & $\begin{array}{l}\text { pJW1 with Type V-A pseudo-CRISPR } \\
\text { array for spacer cloning }\end{array}$ & This study \\
\hline pSDM057 & $\begin{array}{l}\text { pJW1 with Type VI-A (LseCas13a) } \\
\text { pseudo-CRISPR array for spacer } \\
\text { cloning }\end{array}$ & This study \\
\hline pSDM070 & $\begin{array}{l}\text { pJW1 with Type VI-A (LshCas13a) } \\
\text { pseudo-CRISPR array for spacer } \\
\text { cloning }\end{array}$ & This study \\
\hline
\end{tabular}

508 Supplemental Table 3. Oligonucleotides, g-blocks, crRNAs 


\begin{tabular}{|c|c|c|}
\hline I-C_Bsal_for & $\begin{array}{l}\text { gatccGTCGCGCCCCGCACGGGCGCGTGGATTG } \\
\text { AAACgagaccTCTCTGGACAAAggtctc } \\
\text { GTCGCGCCCCGCACGGGCGCGTGGATTGAAAC } \\
\text { a }\end{array}$ & $\begin{array}{l}\text { Underlined regions } \\
\text { indicate location of } \\
\text { Bsal sites }\end{array}$ \\
\hline I-C_Bsal_rev & $\begin{array}{l}\text { agcttGTTTCAATCCACGCGCCCGTGCGGGGCGC } \\
\text { GACgagaccTTTGTCCAGAGAggtctcGTTTCAATCC } \\
\text { ACGCGCCCGTGCGGGGCGCGACg }\end{array}$ & $\begin{array}{l}\text { Underlined regions } \\
\text { indicate location of } \\
\text { Bsal sites }\end{array}$ \\
\hline $\begin{array}{l}\text { sgRNA scaffold } \\
\text { sequence }\end{array}$ & $\begin{array}{l}\text { ccatagagaccACGTACGTACggtctcAGTTTTAGAGC } \\
\text { TAGAAATAGCAAGTTAAAATAAGGCTAGTCCGT } \\
\text { TATCAACTTGAAAAAGTGGCACCGAGTCGGTGC } \\
\text { TTTTTTT }\end{array}$ & $\begin{array}{l}\text { Underlined regions } \\
\text { indicate location of } \\
\text { Bsal sites }\end{array}$ \\
\hline p30T-gRNA_Bsal & ctctactgtttctccatccatagagaccacgtacgtacg & \\
\hline $\begin{array}{l}\text { P30T- } \\
\text { gRNA_Bsal_rev }\end{array}$ & gcccaaaaaaacgggtccgggcaggataggtgaag & \\
\hline gRNA_Bsal-p30T & atggagaaacagtagagagttgc & \\
\hline $\begin{array}{l}\text { gRNA_Bsal-p30T- } \\
\text { rev }\end{array}$ & acccgtttttttgggctag & \\
\hline Cpf1_crRNA_top & $\begin{array}{l}\text { catgaaatttctactgtttgtagatGgagaccTCTCTGGACAAA } \\
\text { ggtctcGaaatttctactgtttgtagat }\end{array}$ & $\begin{array}{l}\text { Underlined regions } \\
\text { indicate location of } \\
\text { Bsal sites }\end{array}$ \\
\hline $\begin{array}{l}\text { Cpf1_crRNA_botto } \\
\text { m }\end{array}$ & $\begin{array}{l}\text { agctatctacaaacagtagaaatttCgagacc TTTGTCCAGA } \\
\text { GAggtctcCatctacaaacagtagaaatt }\end{array}$ & $\begin{array}{l}\text { Underlined regions } \\
\text { indicate location of } \\
\text { Bsal sites }\end{array}$ \\
\hline tracrRNA-FOR & $\begin{array}{l}\text { gaaattaatacgactcaactatagaaaacagcatagcaagttaaaat } \\
\text { a }\end{array}$ & $\begin{array}{l}\text { For T7 RNAP in } \\
\text { vitro rxn }\end{array}$ \\
\hline tracrRNA-REV & aaaaaaagcacccgactcggtgccac & $\begin{array}{l}\text { For T7 RNAP in } \\
\text { vitro rxn }\end{array}$ \\
\hline PTn7R & cacagcataactggactgaatttc & Ref. 39 \\
\hline PglmS-down & gcacatcgggcgacgtgctctc & Ref. 39 \\
\hline $\begin{array}{l}\text { LseCas13a crRNA } \\
\text { Top }\end{array}$ & $\begin{array}{l}\text { catggggtaagagactacctctatatgaaagaggactaaaaccgag } \\
\text { accacgtacgtacggtctccggtaagagactacctctatatgaaaga } \\
\text { ggactaaaacg }\end{array}$ & $\begin{array}{l}\text { Underlined regions } \\
\text { indicate location of } \\
\text { Bsal sites }\end{array}$ \\
\hline $\begin{array}{l}\text { LseCas13a crRNA } \\
\text { Bottom }\end{array}$ & $\begin{array}{l}\text { aattcgttttagtcctctttcatatagaggtagtctcttaccggagaccgta } \\
\text { cgtacgtggtctcggtttagtcctctttcatatagaggtagtctcttaccc }\end{array}$ & $\begin{array}{l}\text { Underlined regions } \\
\text { indicate location of } \\
\text { Bsal sites }\end{array}$ \\
\hline $\begin{array}{l}\text { LshCas13a crRNA } \\
\text { Top }\end{array}$ & $\begin{array}{l}\text { catggggatttagaccaccccaatatcgaaggggactaaaaccgag } \\
\text { accacgtacgtacggtctccggatttagaccaccccaatatcgaagg } \\
\text { ggactaaaacg }\end{array}$ & $\begin{array}{l}\text { Underlined regions } \\
\text { indicate location of } \\
\text { Bsal sites }\end{array}$ \\
\hline $\begin{array}{l}\text { LshCas13a crRNA } \\
\text { Bottom }\end{array}$ & $\begin{array}{l}\text { aattcgttttagtccccttcgatattggggtggtctaaatccggagaccgt } \\
\text { acgtacgtggtctcggtttagtccccttcgatattggggtggtctaaatc } \\
\text { cc }\end{array}$ & $\begin{array}{l}\text { Underlined regions } \\
\text { indicate location of } \\
\text { Bsal sites }\end{array}$ \\
\hline \multicolumn{3}{|l|}{$\begin{array}{l}\text { Alt-R CRISPR- } \\
\text { Cas } 9 \text { crRNA }\end{array}$} \\
\hline $\mathrm{KZ}(\mathrm{IV})-1$ & $\begin{array}{l}\text { rGrArArUrCrUrGrCrUrArArUrArArGrGrU } \\
\text { rUrCrArGrUrUrUrUrArGrArGrCrUrArUrGrC rU }\end{array}$ & Pos. 158,649 \\
\hline
\end{tabular}


509

\begin{tabular}{|l|l|l|}
\hline KZ (IV) - 2 & $\begin{array}{l}\text { rUrCrArCrCrArCrGrCrArUrUrArCrArUr } \\
\text { rCrArGrGrUrUrUrUrArGrArGrCrUrArUrGrC rU }\end{array}$ & Pos. 168,715 \\
\hline DMS3m (IV) - 1 & $\begin{array}{l}\text { rGrCrC rGrArC rArUrU rUrUrC rCrArG rUrUrG } \\
\text { rGrCrG rUrUrU rUrArG rArGrC rUrArU rGrCrU }\end{array}$ & Pos. 17,751 \\
\hline DMS3m (IV) - 2 & $\begin{array}{l}\text { rUrCrA rCrGrA rCrGrA rCrCrC rArGrA rArGrC } \\
\text { rGrUrG rUrUrU rUrArG rArGrC rUrArU rGrCrU }\end{array}$ & Pos. 28,033 \\
\hline
\end{tabular}


bioRxiv preprint dol: https://doi.org/10.1101/370791; this version posted July 17, 2018. The copyright holder for this preprint (which was not certified by peer review) is the author/funder, who has granted bioRxiv a license to display the preprint in perpetuity. It is made available under aCC-BY-NC 4.0 International license.

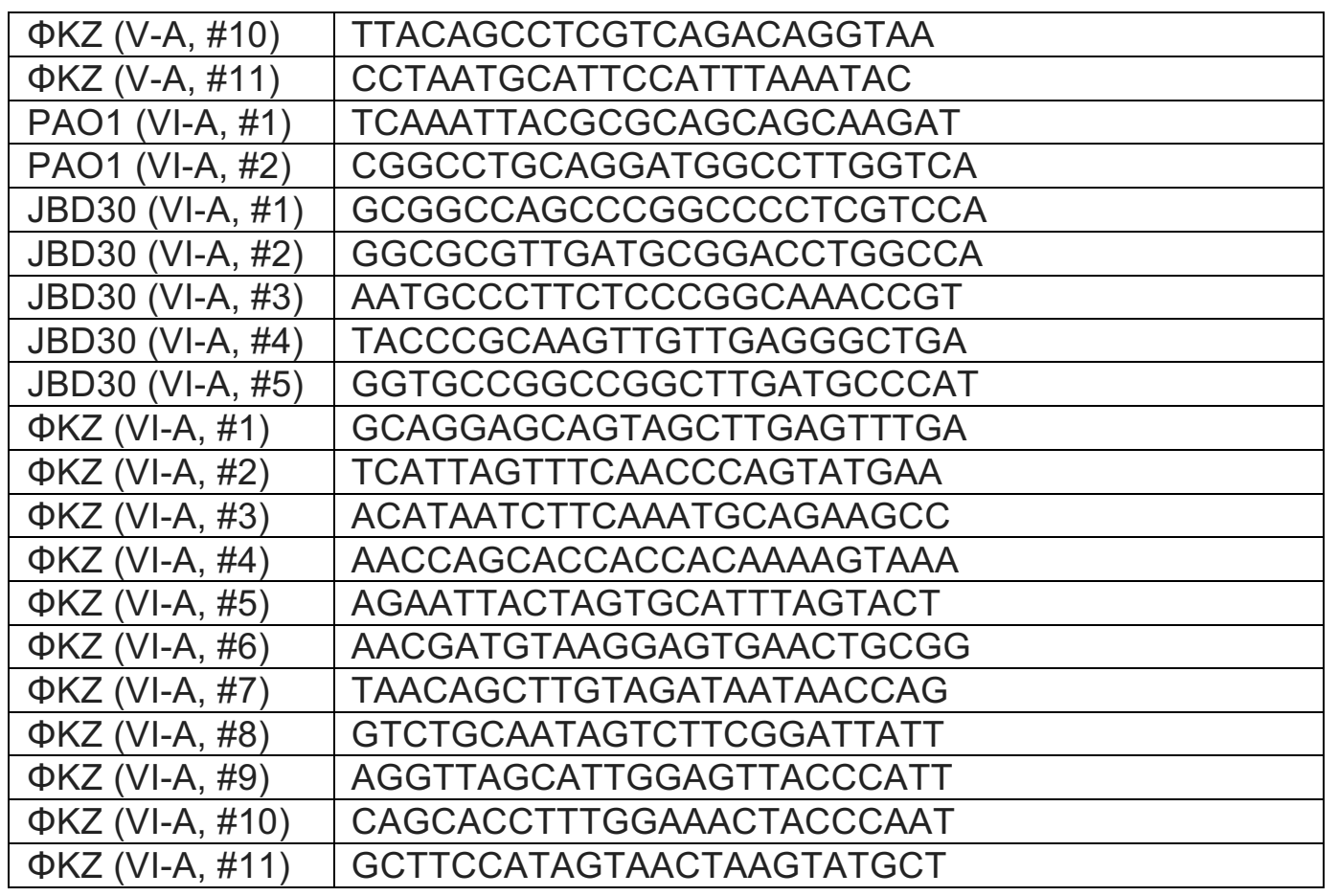

511

512 